\title{
NORMES INTERNATIONALES DE JUSTICE ET GLOBALISATION DE L'ETHIQUE
}

Cathérine Audard*

SÍNTESE - O artigo procura mostrar que sem uma comunidade civil democratizante de justificação, em lugar do atual sistema internacional, as normas da justiça global não passam de uma ficção, uma mera expressão do imperialismo cultural e político, um instrumento de controle e dominação dos povos em escala mundial, segundo um modelo colonizador ampliado que torna as declarações dos direitos humanos inoperantes. PALAVRAS-CHAVE - Direitos humanos. Ética Habermas. Justiça global. Normas internacionais. Rawls.
ABSTRACT - The article seeks to show that without a civil, democratizing community of justification, replacing the current international system, the norms of global justice remain a fiction, a mere expression of cultural and political imperialism, an instrument of control and domination of the peoples worldwide, according to an enlarged colonizing model which render the declarations of human rights inoperative.

KEY WORDS - Ethics. Global justice. Habermas. Human rights. International norms. Rawls.

Je voudrais examiner ici quelques-unes des difficultés que présente la notion de normes internationales de justice. Une des critiques les plus graves est qu'elles seraient la pure et simple expression d'un impérialisme culturel et politique, un instrument de contrôle et de domination des populations à l'échelle du globe, selon un modèle colonisateur élargi. ${ }^{1}$ Inversement, en consultant, par exemple, des documents défendant un "droit au développement " que certains pays voudraient faire inscrire dans la Déclaration universelle des droits de l'homme de 1948, parmi les droits imprescriptibles de l'humanité, on est frappé par l'usage " essentialiste " et non critique de la référence aux droits de l'homme et à une justice internationale.

Entre ces deux extrêmes, y a-t-il de la place pour une interprétation différente ? Peut-on "prendre les droits au sérieux », selon la formule célèbre du philosophe et juriste américain Ronald Dworkin, sans transformer la Déclaration de 1948 en dogme, ce qui aurait des conséquences politiques et culturelles dommageables ? Peut-on voir dans cette référence à des normes internationales de justice une aspiration proprement éthique ou n'est-ce qu'une nécessité politique? Som-

Doutora. Professora do Departmento de Filosofia da London School of Economics.

Hardt et Negri, 2000, B. Hindess, 2002.

\begin{tabular}{|l|l|l|l|l|l|}
\hline VERITAS & Porto Alegre & v. 50 & n. 1 & Março 2005 & p. 23-39 \\
\hline
\end{tabular}


mes-nous en route vers une éthique globale, ou l'internationalisation des normes n'est-elle que le résultat des rapports de force au sein de la communauté des nations?

Existe-t-il un socle éthique commun à l'humanité ? La crise du droit naturel qui s'ouvre au XVIIIème siècle avec Hume et son analyse de la justice a rendu désormais impossible de se référer à une loi naturelle, commune à l'humanité, pour s'opposer aux abus et aux crimes commis par le pouvoir politique, pour fonder ce que Locke appelle «le droit de résistance à l'oppression $\|^{2}$ Le monde moderne a trouvé dans des Déclarations des droits de l'homme (1789, 1948, 1976 Helsinki, 1992 Convention européenne) au statut conceptuel et politique incertain, un substitut à la fois indispensable et problématique au droit naturel, divin ou rationnel. La question d'un "fondement » des droits de l'homme est alors passée au premier plan des préoccupations. Or, pour des raisons que j'examinerai ici, il est impossible de fournir un fondement de ce genre et si l'on en trouvait un, on pourrait suspecter sa fonction dans l'ordre politique international. Sommes-nous alors voués au relativisme culturel et moral ? Un accord sur des normes internationales de justice est-il impossible ?

J'examinerai en premier lieu (I) les confusions qui entourent la notion de normes internationales de justice. Puis je montrerai (II) comment ces normes ne peuvent acquérir de contenu éthique et de légitimité que par une procédure publique de justification et de validation à laquelle tous ceux qui sont concernés participent également. La demande de normes internationales de justice passe donc par la démocratisation des institutions internationales (III).

\section{Normes internationales de justice ou impérialisme ?}

Mais, tout d'abord, que faut-il entendre par « normes internationales de justice »?

Donner une définition préalable à partir de normes internationales déjà existantes reviendrait, comme le note judicieusement Mireille Delmas-Marty ${ }^{3}$, à résoudre le problème avant même de l'avoir posé, à définir l'internationalisation comme seulement ou avant tout une "diffusion spatiale ». Or, l'idée de normes implique quelque chose de bien différent, un "partage de sens », un horizon du souhaitable, du désirable qui va au-delà d'un processus politique, économique et juridique observable. En un sens, les normes internationales de justice n'existent pas plus que la justice elle-même n'existe, elles constituent plutôt un projet pour l'humanité, un idéal régulateur de l'action humaine et donc, en ce sens, leur définition, leur élaboration ne peuvent être qu'un travail collectif.

David Hume, Traité de la nature humaine, livre III (1740) et John Locke, Second Traité du gouvernement civil, chapitre XIX (1689).

M. Delmas-Marty, Les treois défis d'un droit mondial, p. 14. 


\subsection{Internationalisation ou globalisation des normes?}

La difficulté principale est que la notion cherche à articuler l'universel au sens d'un devoir, d'un impératif moral catégorique, valable pour tous sans exception, et l'international, au sens d'un élargissement de l'espace où des règlements et des accords juridiques, politiques et économiques entre nations sont reconnus et validés. Mais cette définition est une source de confusions.

Tout d'abord, elle suppose que l'objet du souci éthique, le destinataire de la norme est la nation ou l'État, et non pas les individus concernés. Or les États non seulement n'ont pas de statut moral, mais ils sont la source des manquements éthiques les plus graves qui soient à l'égard des êtres humains, comme, par exemple, les crimes contre l'humanité. Il nous faudra donc distinguer entre une internationalisation de l'éthique, dans laquelle les normes propres à un pays ou une culture sont étendues au système des nations, et une universalisation ou une globalisation de l'éthique dans laquelle les destinataires comme les producteurs des normes sont les personnes elles-mêmes.

Cette confusion politique se double d'une confusion conceptuelle. L'idée de normes internationales ignore la tension entre le fait et le droit, entre la norme comme conduite majoritaire, observable comme telle, et la norme comme ce qui s'impose aux conduites pour les transformer. Elle semble vouloir réduire ou ignorer la tension entre ce qui est et ce qui devrait être. Par exemple, l'espoir placé dans la Déclaration universelle des droits de l'homme de 1948 pour dénoncer les injustices et punir les crimes commis contre l'humanité, au Cambodge, au Rwanda, en Bosnie ou au Kosovo s'est incarné dans une internationalisation des conflits et du droit et dans une foi dans un nouveau Tribunal pénal international, qui pourchasserait partout et à jamais les coupables. L'internationalisation de la justice semble témoigner de sa moralisation. Mais ne soyons pas dupes. La simple internationalisation ne moralise pas les actions.

On ne peut cependant en rester à un hiatus entre le droit et les pratique politiques, d'un côté, l'éthique de l'autre. La norme est bien ce qui est observable, ce qui décrit le comportement habituel, le plus répandu, prévisible, commun. Allons plus loin. Sans impact sur la réalité, sur les faits, les normes n'ont pas de sens et sont des prescriptions creuses, de simples "idéaux de l'imagination ", selon la formule hégélienne. ${ }^{4}$ Un des faits historiques les plus considérables du XXème siècle a été la transformation des droits de l'homme en véritables droit positifs, ratifiés par les nations et inscrits dans leur constitution et les obligeant à remanier leur propre système du droit, comme dans le cas des pays de l'Union européenne et de la Convention européenne des droits de l'homme. Cette transformation indique clairement que la distinction entre le fait et le droit est interne aux normes de justice elles-mêmes qui, à la différence de simples valeurs posées ou désirées, doivent trouver leur incarnation dans une réalité juridique et un code coercitif de règlements judiciaires, économiques et politiques.

Hegel, La raison dans l'histoire, Introduction, II ${ }^{\mathrm{èm}}$ Partie. 


\subsection{L'illusion rationaliste}

D'autre part, sur le plan conceptuel, l'idée de normes internationales risque d'être victime d'une illusion rationaliste que la critique culturaliste contemporaine a dénoncée à juste titre. Le raisonnement qui a consisté à parler des droits de l'humanité pour fonder la notion juridique de crime contre l'humanité, par exemple, ne peut être valable pour toutes les cultures, s'appliquer à tous les droits énoncés dans la Déclaration. On peut parler d'une nouvelle forme d'intolérance si toute déviation par rapport à la norme universelle est considérée comme une erreur ou une faute amenée à disparaitre, si le caractère pluraliste des sociétés est nié. Toute une philosophie rationaliste de l'unité de l'humanité sous-tend donc ces Déclarations, ce qui doit conduire à s'en méfier. L'idée même que ces droits humains doivent être fondés philosophiquement est contradictoire puisque s'ils sont universels, ils n'ont pas besoin de fondement pour être reconnus et appliqués, et qu'aucune philosophie particulière ne saurait les fonder sans faire la preuve de leur caractère local et relatif à une conception particulière et dominante du Bien.

\subsection{Définition provisoire}

Il vaut donc mieux définir les normes en termes d'objectifs et non en termes impératifs, insister sur leur dimension évaluative ou corrective d'états de choses inacceptables comme le fait Martha Nussbaum. ${ }^{5}$ Des objectifs tels que l'éradication de la pauvreté, objectif que se sont donné nombre d'organisations internationales et de gouvernements dans leur politique d'aide au développement, le respect de l'environnement, la protection de l'intégrité de la personne, le droit à l'immigration, le droit d'asile, la reconnaissance culturelle des droits des minorités, pour prendre quelques exemples de normes de mieux en mieux acceptées, dépendent tous d'une conception de la justice sur laquelle les sociétés démocratiques semblent en accord.

Celle-ci inclut également une conception de ce que serait une juste distribution des biens publics qui permettrait d'atteindre ces objectifs. Enfin, une telle définition suppose une conception du bien, du " bien démocratique $\|^{6}$ qui sous-tend la Déclaration de 1948 et ses développements récents et sur lequel un accord devrait être trouvé. Tout cela demande à être discuté dans des délibérations publiques à l'échelle globale de manière à ce que la signification précise de ces objectifs et des transformations qu'ils impliquent puisse être traduite dans les différents idiomes des " nations " qui composent le "village global ». Mais s'arrêter à un consensus entre nations ne suffit pas. L'objectif suprême est d'impliquer les populations elles-mêmes, les individus concernés. On pourra alors parler de normes " cosmopolites » ou " globales " afin de donner à l'éthique un champ qui ne soit pas limité par les nations ou le système de leurs relations. L'extension des normes dans

Martha Nussbaum, 2000, p. 39-41

David Held, 1995, chapitre 9. 
l'espace international à toute l'humanité ne garantit pas l'égalité des personnes ou des États. Il faut y ajouter une conception des biens publics humains qui échappent à la juridiction nationale, aux États. Le cosmopolitisme, par contraste avec l'internationalisme, est une doctrine qui ne conjugue pas l'éthique avec l'identité politique ou culturelle, mais qui est concernée par la justice et les droits des êtres humains en tant que personnes. Il cherche à comparer et à évaluer des situations différentes et déficientes, fonctionnant comme une sorte de tribunal indépendant des États, ayant une dimension globale et pas seulement internationale.

\section{Le nouveau système international}

Or on ne peut répondre à toutes ces questions sans avoir clarifié au préalable la nature du contexte international de la justice, ce que David Hume appelle « les circonstances de la justice ॥. $^{\text {? }}$

\section{1 « Les circonstances de la justice »}

La question de la justice ne se pose que s'il y a conflit entre des agents au sujet de biens, rares et précieux, que tous désirent avec une égale intensité. La question de la justice ne se pose pas si le conflit est inégal et peut être réglé uniquement par la force. Le résultat du conflit n'aura aucune prétention à être « juste ». Elle ne se pose pas non plus si les agents ne sont pas réellement en conflit, s'ils sont totalement rationnels et se répartissent les biens sans discussion. La question de la justice ne se pose que là où des règles, des lois ou des procédures, doivent être trouvées pour donner à chaque plaignant son dû de manière satisfaisante. Ce contexte est donc à la fois objectif, constitué par les biens disputés, et subjectif, constitué par les acteurs du conflit.

Voyons tout d'abord rapidement la nature des biens disputés.

Une définition des normes internationales inclut une réflexion sur ce qui doit être redistribué de manière équitable à l'échelle internationale: la santé, la sécurité, l'éducation, les ressources naturelles, le savoir, un environnement viable, les revenus, etc. Ces conditions qui permettent l'accès à notre commune humanité ainsi que son développement peuvent-elles être décrites de manière satisfaisante en termes de biens ? Ces biens sont-ils définis par ce que certains appellent les besoins de base de l'humanité, sans qu'un accord sur ce minimum puisse être garanti ? Ou faut-il les définir à partir des préférences des consommateurs, là encore sans être certains que les préférences aient une valeur objective et puissent être comparées entre elles? S'agit-il, comme pour John Rawls ${ }^{8}$, de «biens premiers ", c'est-à-dire de tout ce dont un individu a besoin pour promouvoir et réaliser son plan de vie et participer pleinement à la prise de décisions collectives

Hume, Traité de la nature humaine, livre III, p. 95-97.

John Rawls, Théorie de la justice, p.93 : "Posons que les différents biens premiers à la disposition de la société sont les droits, les libertés et les possibilités offertes à l'individu, les revenus et la richesse.. .et le respect de soi-même. » 
engageant son bien-être et son avenir ? Cette approche fondée sur les ressources disponibles, non sur une définition discutable de la "nature humaine " ou de " l'homme générique au sens de Marx, a l'immense mérite de ne pas s'arrêter à une liste de droits formels, mais d'inclure leurs conditions de réalisation sociale et économique. Cette approche a été récemment renouvelée par l'économiste Amartya Sen $^{9}$ grâce à une approche éthique qui définit les bien premiers non comme des biens ou des ressources, au sens habituel d'objets ou de moyens de consommation, mais comme des " capabilités ", c'est-à-dire tout ce dont un être humain a besoin pour s'épanouir selon ses propres critères, pour son fonctionnement optimal.

Le contexte subjectif concerne, lui, les acteurs en présence sur la scène internationale.

Quelques remarques préliminaires s'imposent ici. Les acteurs sont-ils les États ou les individus? Tout le débat tourne autour de ces deux conceptions. ${ }^{10}$ Soit, pour des raisons de réalisme politique comme de respect du pluralisme, on reste dans le cadre traditionnel du droit international public, du "droit des gens ", jus gentium, même en l'améliorant et les acteurs sont les États souverains et leurs relations. C'est le choix, par exemple, de John Rawls dans sa théorie de la justice appliquée au contexte international. ${ }^{11}$ Mais, comme nous le verrons, il devient difficile dans ce contexte de parler de globalisation de l'éthique. Soit on opte pour un cadre cosmopolite, au sens de Kant, et les acteurs sont les individus et nioon plus les États. Plusieurs arguments penchent pour cette dernière option.

Il est extrêmement difficile de définir le statut éthique d'une nation, d'un État, abstraction faite de celui des personnes qui le constituent. Or, si l'on regarde la situation des droits de l'homme et des droits des citoyens dans la plupart des pays membres de la communauté internationale, on voit à quel point il est dangereux de prendre comme unité de mesure les États et non pas les individus. Les États sont une trop grande menace pour les droits des individus d'une manière générale. L'existence d'institutions comme le gouvernement représentatif, l'État de droit, (rule of law) peut certes être encourageante, mais en définitive, est-ce plus qu'une condition nécessaire de l'éthique globale? Il est douteux que les acteurs concernés par les normes internationales soient au premier chef les États, et non pas les populations elles-mêmes.

9 Amartya Sen, Éthique et économie, p.218 : "Dans l'évaluation de la justice qui se fonde sur la capabilité, les exigences des individus ne sont pas évaluées en fonction des ressources ou des biens premiers qu'ils possèdent, mais d'après la liberté qu'ont effectivement les individus de choisir entre différents modes de vie auxquels ils peuvent tenir. C'est cette liberté réelle qu'on désigne par la « capabilité » d'accomplir plusieurs combinaisons possibles de modes de fonctionnement. "

${ }^{10}$ Voir par exemple, Thomas Pogge, Realizing Rawls, chapitre 6 et Charles Beitz, "Rawls' Law of Peoples ", p.680 : "C'est une chose de soutenir qu'une Société des nations globale est un objectif souhaitable du changement politique et social, et une tout autre de soutenir que les principes de l'éthique internationale doivent être fondés sur les intérêts légitimes des peuples plutôt que des personnes. "

11 John Rawls, Le droit des gens 
Si l'on reste dans le cadre du droit international public, c'est essentiellement au nom, non pas de la justice, mais de la stabilité des relations internationales. C'est donc une justification politique et non pas morale du respect des droits de l'homme. Des considérations cosmopolites n'ont pas leur place quand l'unité de représentation est constituée par les États, pas les individus.

\subsection{Le système " post-westphalien »}

Or quelle est la situation politique actuelle ? Les acteurs des relations internationales ont été, depuis la paix de Westphalie (1648), les États souverains qui ont progressivement pris la place des empires. Ces États territoriaux refusent toute ingérence dans leur domaine de juridiction, tout défi à leur autorité, et ne négocient entre eux que sur un pied d'égalité, exacerbant les conflits et intensifiant le recours à la guerre comme solution puisque aucune instance supérieure n'est dorénavant acceptable par tous les acteurs comme l'était, par exemple, l'autorité religieuse du Pape pour la Chrétienté médiévale.

Mais depuis 1945, ce système westphalien a été sérieusement ébranlé. Un nouveau système régulateur des échanges internationaux s'est mis en place sur tout le globe à la suite des désastres de la seconde guerre mondiale. Couvrant à la fois les préoccupations politiques et économiques, ce système d'institutions internationales (FMI, Banque mondiale, OMC, Nations Unies, GATT, OCDE, OTAN, etc.), en réalité, ne fait que continuer une coordination entre États qui existait déjà, mais qui était plutôt politique, le système des Alliances, ou culturel. Il l'a étendu à tous les aspects de la souveraineté, la création de l'Union européenne étant l'exemple le plus tangible de cette évolution. Il a surtout créé de nouvelles inégalités en détruisant la fiction de relations entre États souverains et égaux. Enfin, en affaiblissant directement ou indirectement le rôle des États, surtout dans les pays les plus démunis, il a créé une demande d'aide et d'intervention internationales qui s'est avérée impossible à satisfaire.

Une nouvelle hiérarchie entre États pauvres et demandeurs d'aide, et États donataires en est résultée, répliquant et amplifiant les différences sociales et économiques de chaque pays sur toute la planète. En même temps, la demande que les droits les plus importants des citoyens du monde soient appliqués est devenue beaucoup plus urgente en raison de cette extension territoriale et de cette visibilité croissante. Les plus pauvres dans un pays le sont encore davantage si ce pays est au bas de la liste des États selon leur PNB. Ils dépendent pour leur survie non seulement de leur propre gouvernement, qui souvent abandonne ses responsabilités en raison du nouveau système d'aide et d'intervention, mais également et même surtout de la communauté internationale elle-même par le biais des institutions internationales et de l'opinion publique. D'une certaine manière, le nouveau système a aggravé la situation en ouvrant ainsi des possibilités nouvelles, mais totalement en dehors du contrôle des populations. 


\subsection{La promesse de démocratie}

Parmi ces possibilités nouvelles, la première place est certainement celle de la promesse de démocratie.

L'une des constantes des politiques de développement contemporaines est justement que la démocratisation des régimes politiques est un objectif prioritaire. L'aide économique est suspendue à la réforme des institutions politiques, à la " bonne gouvernance ", pour reprendre l'expression officielle. À côté de la santé et de l'éducation, la réforme des institutions politiques, la lutte contre la corruption, le développement d'une administration intègre et compétente, sont devenus les leitmotivs des pays donataires. On ne peut certes que se réjouir de cette transformation du langage des technocrates et des politiques.

Mais ici plusieurs remarques sont nécessaires. Tout d'abord, il faut noter une tonalité normative qui rappelle l'entreprise coloniale. La justification de l'entreprise coloniale était bien souvent d'avoir une mission " civilisatrice ». De même on retrouve dans le discours de l'aide internationale et des échanges internationaux la référence à une " norme de civilisation » dont les pays les plus puissants et riches assumeraient la responsabilité vis-à-vis des populations les plus démunies. Que cette nouvelle norme soit la démocratie est sans doute un progrès, mais cela ne change rien au contrôle exercé sur les populations. Le critère qui permet de sortir du cycle de la pauvreté est dorénavant d'être capable de participer aux échanges économiques internationaux et de suivre les recommandations des organismes internationaux de contrôle. Le discours de l'amélioration morale de l'humanité est peut-être ainsi devenu plus acceptable, mais le contrôle est d'autant plus insupportable qu'il est imposé au nom de la défense des droits de l'homme et de l'autonomie des citoyens. La critique ne porte pas sur le contenu de cette norme de civilisation, mais sur la manière dont elle est imposée.

D'autre part, dans aucun pays développé, l'application de l'État de droit, le respect des droits de l'homme et la participation des citoyens, le processus de démocratisation dans tous ses aspects donc, n'ont été imposés de l'extérieur. Ils ont été le résultat de conquêtes politiques par les citoyens qui ont supposé des transformations sociales et économiques en profondeur. Or la brutalité du processus actuel d'imposition de normes internationales de justice est contraire à ses objectifs. Même si les programmes de développement insistent de plus en plus et à juste titre sur la participation des populations concernées par ces programmes, la démocratisation de ce processus est encore loin d'être réalisée, et même d'être comprise dans sa nature profonde : la solidarité entre les droits de l'homme, civils, politiques, économiques et culturels, et la situation économique et sociale.

Le problème le plus crucial est donc celui de l'inégalité des acteurs, individuels et collectifs, accentuée par l'apparition d'organismes internationaux qui se substituent de plus en plus à l'État et à ses responsabilités. L'internationalisation du droit et du développement, bien loin de faire avancer sur la voie de la justice, a créé les conditions d'une inégalité encore plus injuste entre les peuples, encore plus visible et insupportable. 
On ne peut légitimer les normes internationales qu'en démocratisant les institutions et le procédures de prise de décisions. Tant que l'on aura un système des nations hiérarchique et autoritaire, un pouvoir sans limites, unilatéral, et qui ne rend de comptes à aucun électorat, on ne pourra parler de normes de justice. Si la structure qui prétend aider à la justice est elle-même injuste et ne respecte pas l'autonomie des agents concernés, personnes ou nations, elle ne peut conduire à des résultats acceptables.

\section{La démocratisation des normes internationales}

Comment alors procéder et concilier justice et autonomie des personnes ? Dans son essai de 1795, La paix perpétuelle, Kant nous donne une indication tout à fait claire. "C'est la volonté universelle, écrit-il, donnée a priori (dans un peuple ou dans le rapport mutuel des différents peuples) qui est la seule à déterminer ce qui est de droit parmi les hommes. ${ }^{12}$ En d'autres termes, ce sont les individus concernés eux-mêmes qui doivent déterminer ces principes de justice conformément à la définition de la liberté comme autonomie, comme obéissance à la loi qu'on s'est soi-même donnée, principe de base de tout régime républicain qui, pour Kant, devrait être étendu au globe. Faisant la critique du « moraliste politique qui fabrique une morale à la convenance de l'homme d'État $"{ }^{13}$ il nous invite à méditer sur le fait que sans transformation des régimes politiques et de leurs relations, il ne peut exister de paix perpétuelle entre les nations, seulement une paix impériale. Démocratiser le processus d'élaboration et d'application des normes internationales de justice est donc le seul moyen de leur donner une légitimité morale, de globaliser l'éthique.

Mais il est impensable de faire dépendre la légitimité des normes de la seule volonté morale des individus. En termes kantiens, la "phénoménalisation » de la liberté humaine suppose l'intervention du droit comme médiation entre la nature, au sens des inégalités naturelles entre les hommes et les nations, et la morale. «Cette réunion de la volonté de tous peut... être en même temps, d'après le mécanisme de la nature, la cause permettant de provoquer l'effet visé et de rendre effectif le concept de droit. "14 Aussi bien au plan national qu'international, donc, le niveau crucial est celui de la production et de l'application des normes juridiques dans des conditions démocratiques qui en garantissent la légitimité. Tel est le sens de la réflexion de Jürgen Habermas dans Droit et démocratie. Pour donner un sens concret au projet kantien, il faut comprendre la volonté universelle non comme donnée a priori, mais comme le résultat d'une procédure de délibération. C'est également le point de vue défendu par John Rawls dans Théorie de la justice. Des normes internationales de justice ne sont valables que si elles sont le

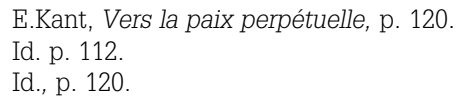


résultat d'une procédure de justification dans laquelle tous ceux qui sont concernés sont des partenaires sur un pied d'égalité.

\subsection{Justice et justification}

Ce qui caractérise l'univers culturel des démocraties contemporaines, c'est d'être entrées dans un monde "post-métaphysique » à la suite d'une révolution qui date de Hume. La justice, pour Hume, est " une vertu artificielle " qui ne repose pas sur une loi naturelle, divine ou religieuse. "Le sens de la justice et de l'injustice ne vient pas de la nature, mais résulte artificiellement, quoique nécessairement, de l'éducation et des conventions humaines. " ${ }^{15}$ Mais, " bien que les lois de la justice soient artificielles, elles ne sont pas arbitraires. ${ }^{16}$ Le problème de la justice est donc devenu celui de sa justification, de l'acceptabilité rationnelle de ses principes par ceux-là mêmes qui auront à les appliquer.

En d'autres termes, le substitut de la loi naturelle se trouve dans une nouvelle conception de la subjectivité humaine, capable de parvenir à un consensus sur des normes universelles, et structurée autant par l'amour de soi et la partialité que par la sympathie, la capacité à coopérer et à délibérer avec autrui. Lorsque nous essayons de construire de manière démocratique une théorie de la justice internationale, nous sommes donc confrontés à deux séries de difficultés. D'une part, le problème philosophique est celui d'un fondement des normes dans un consensus qui ne dépend pas d'un droit naturel transcendant et qui n'est pas non plus ethnocentrique, imposant la même norme à tous. D'autre part, le problème politique est celui de lutter contre l'hégémonie, dans la production des normes, des structures internationales, hiérarchiques et autoritaires.

Si la procédure de justification des normes est ouverte à tous, en tant que citoyens du monde, si elle est véritablement, pour reprendre une formule kantienne, à la fois " universelle et subjective ", alors nos deux difficultés peuvent être surmontées.

Une première manière de procéder consisterait à utiliser des critères déjà présents dans la morale ordinaire, dominante, de manière à rallier une majorité. Mais ce faisant, on nuirait aux populations minoritaires et le principe démocratique d'égalité des nations ne serait pas appliqué. De plus, ces principes ne résisteraient pas au critère de publicité sur lequel Kant insiste dans Vers la paix perpétuelle et qui demande de pouvoir « parler librement et publiquement des maximes universelles... s'exprimer librement. " ${ }^{17}$ Seul l'usage de la force permettrait d'atteindre le consensus sur ces normes ou règles majoritaires.

Une seconde manière chercherait ce qu'il y a de commun aux hommes et qui les unit, alors que les croyances morales, les conceptions du bien, les divisent. L'utilitarisme affirme un principe universel de ce genre : le bonheur et sa maximi-

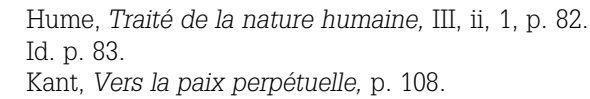


sation sont recherchés par tous, donc ils peuvent servir de base à des principes de répartition universellement acceptables si l'on montre que leurs conséquences augmentent la quantité de satisfaction de tous ou, du moins, du plus grand nombre. Mais, là encore, le pluralisme démocratique n'est pas respecté pas plus que le caractère distinct de personnes qui sont loin d'accepter mécaniquement que le bien-être soit le seul bien.

Pour surmonter ces difficultés, John Rawls avance une série d'arguments complexes qui peuvent être adaptés ou étendus à la justice internationale. ${ }^{18}$ Il cherche à dériver les principes de justice de la seule rationalité instrumentale, sans faire intervenir de prémisses éthiques. Il part d'une situation initiale de choix rationnel qui, si elle est équitable, donnera un résultat lui-même équitable : les principes ne sont pas des a priori, mais, comme pour Hume, des conséquences de l'interaction entre les acteurs eux-mêmes: on parlera à ce propos de « justice procédurale pure. ${ }^{19}$ Mettons-nous dans la situation hypothétique de choix d'un contrat équitable avec tous nos concitoyens, situation dont les conditions sont l'égalité d'accès à la discussion, la liberté et la publicité des débats. Dans l'ignorance de notre situation personnelle dans la distribution des ressources (le célèbre « voile d'ignorance »), nous choisirions nécessairement, dit Rawls, trois principes de justice : (1) le principe d'égale liberté pour tous, lexicalement prioritaire, (2) le principe de juste égalité des chances, (3) complété par le principe de différence ou de justification des inégalités utiles aux plus défavorisés.

Un aspect de la procédure publique de justification selon Rawls mérite de s'y arrêter, car il a des conséquences pour la justice internationale.

La constitution d'une communauté de justification seule, selon lui, donne sa légitimité aux principes communs, quand bien même ils diffèrent de notre conception morale ordinaire. «Étant conçue pour réconcilier grâce à la raison, la justification a son point de départ dans ce que tous les partenaires de la discussion ont en commun. ${ }^{20}$ Cela suppose qu'ils mettent de côté ce qui les sépare pour se mettre d'accord sur les principes de leur coopération. Cette exigence d'impartialité est représentée, chez Rawls, par la fiction du contrat social équitable, c'est-à-dire d'une position originelle où les contractants choisissent les principes qui gouverneront leur association politique en étant placés derrière un « voile d'ignorance ». Que vaudrait en effet des principes que je pourrais modifier en fonction des informations sur mes circonstances sociales, mes mérites et mes talents ou mes handicaps? L'équité suppose que chacun ait des chances raisonnables de bénéficier de la coopération. Comme l'écrit le philosophe américain T.Scanlon, " un test pour savoir si une justification est suffisante consiste à voir si on l'accepterait toujours en étant à la place d'autrui. L'harmonisation des jugements demande la construction d'une forme réellement interpersonnelle de justification qui

18 John Rawls, Théorie de la justice, en particulier §4. Il parle d'une extension à la justice internationale dans le $\S 58$ et dans Le droit de sgens.

9 Id., p. 117.

${ }^{20}$ Id., p. 580. 
soit néanmoins acceptable pour chaque individu. Notre souci de moralité n'est pas seulement instrumental... L'idée d'un accord général ne vient pas simplement d'un besoin de garantir la protection de nos intérêts. Elle est, en un sens plus fondamental, au coeur de ce qu'est la moralité. ॥ $^{21}$ C'est cet argument qui rend plausible le principe de différence ou de protection des plus défavorisés dont on peut dériver un principe d'assistance aux pays les plus pauvres ; ce dernier serait adopté dans l'ignorance où chacun est de la probabilité qu'il occupe la position du plus désavantagé socialement. Est juste ce qui est jugé comme tel à partir d'un point de vue impartial. Ainsi, l'artifice de la position originelle permettrait de définir le juste sans référence ni à des critères externes ni à une conception particulière du bien. Et surtout, sans référence à des propriétés de la personne qui ne sont pas pertinentes pour arriver à une justification comme la race, la couleur de la peau, la différence culturelle, la religion, le genre, etc. Les seules propriétés pertinentes sont celles qui renvoient à des facultés morales : le sens de la justice et la capacité à agir selon une conception du bien. Une telle démarche est donc susceptible de nous conduire vers une globalisation de l'éthique, applicable à tous et reconnue par tous.

\subsection{Délibération et démocratisation de la justice internationale}

Peut-on élargir l'analyse de Rawls, qui ne propose pas une théorie de la démocratie, mais plutôt de la démocratisation des principes constitutifs de l'espace public national, à la démocratisation de la justice internationale ? Comment faut-il comprendre l'exigence de démocratisation, non seulement au niveau des principes, mais au niveau de décisions politiques que l'idéal de justice doit informer ?

Rawls lui-même, dans son effort pour élargir sa théorie et l'appliquer à la justice internationale n'a pas pensé qu'il était possible de démocratiser les relations internationales. Il se limite, dans Le droit des gens, à envisager les principes de justice qui pourraient régir les échanges entre états démocratiques et non démocratiques. Il exclut la possibilité d'universaliser les normes de justice en raison du pluralisme culturel et, surtout, de l'existence d'états « hors la loi ", avec lesquels la coopération est impossible. Il exclut, par exemple, toute possibilité d'exiger un droit des populations à des institutions démocratiques et préfère composer, au nom du réalisme politique, avec ce qu'il appelle des " états décents ", mais non démocratiques $^{22}$. Le problème est qu'il demeure dans le cadre du jus gentium et qu'il ne fait pas des individus eux-mêmes les acteurs de ce processus d'élaboration et de justification de normes.

Pour répondre à notre question initiale et éviter le pessimisme de Rawls, il faut sortir du cadre habituel de la démocratie représentative qui n'a de sens que

\footnotetext{
T. Scanlon " Contractualisme et utilitarisme », in Catherine Audard, Anthologie de l'utilitarisme, vol. III, p. 172.

${ }_{22}$ Rawls, Le droit des gens , p. 63 sur les sociétés hiérarchiques et "bien ordonnées " ou " décentes ".
} 
dans le cadre national. En effet, le système représentatif et parlementaire est insuffisant dans le cadre d'une justice globale, quand il s'agit non pas de prendre des décisions particulières, mais de dégager ensemble des principes et des objectifs pour les actions des gouvernements et des organisations internationales, quand la reconnaissance et le respect pour chacun des acteurs sont aussi importants, si ce n'est plus que les exigences d'efficacité. Il faut donc cesser de comprendre la démocratie en termes purement électoraux, de partis et de négociations entre intérêts, qui n'existent pleinement que dans le cadre national, en présence d'un arbitre qui est l'électorat, pour insister sur le caractère normatif de l'idéal démocratique et la conception de la justice et de la légitimité qui l'anime. Le modèle de démocratie délibérative ${ }^{23}$ que présentent nombre d'auteurs dont le plus important est Habermas, répond exactement à cette demande. De quoi s'agit-il ?

Alors que les démocraties représentatives et parlementaires classiques voient dans le vote et la négociation entre intérêts particuliers le processus politique par excellence, la démocratie délibérative accorde la prépondérance à la délibération publique, soit sous la forme de la "raison publique " des magistrats et des représentants élus de la nation, le forum public politique proprement dit, soit de celle d'une discussion, d'une " conversation » entre citoyens dans l'espace public. On notera que Habermas établit une distinction importante entre espace ou forum public et société civile qui, elle, concerne surtout les échanges et la défense des intérêts personnels ou de groupe. Il s'agit d'abord de démocratiser la société civile, de la transformer en forum public pour que tous les intérêts, et pas seulement ceux des plus puissants ou actifs, soient représentés. C'est sur ce modèle que la société civile internationale pourrait être transformée en incluant tous les citoyens et pas seulement les gouvernements, les organisations internationales, les ONG et autres groupes de pression.

Pour Habermas, la démocratie délibérative est le remède aux désastres et à l'instabilité de la démocratie électorale à condition que nous reconnaissions qu'il n'y a pas d'espoir ultime de réconciliation à la fin de ce débat. Notre adversaire a d'aussi bonnes raisons que nous, et le signe le plus sûr que nous vivons dans une démocratie est que nous devons accepter d'obéir à des décisions avec lesquelles nous ne sommes pas d'accord. Elles sont cependant justifiées et acceptables par ce qu'elles s'appuient sur de " bonnes raisons », des raisons que nous reconnaissons comme telles, non sur la force ou la peur. Ce double idéal de rationalité et d'une normativité propre à la discussion est au cœaur de l'idéal de la démocratie délibérative au sens de Habermas.

La délibération légitime la décision finale aux yeux des participants alors qu'au départ, elle n'était pas nécessairement la meilleure pour chacun. La source de la légitimité n'est pas la volonté pré-déterminée des individus, comme dans le vote majoritaire, mais plutôt le processus de formation de la volonté générale, la

23 Habermas, L'intégration républicaine, 1998, chap. 9 'Trois modèles de démocratie'. On trouvera une bibliographie complète concernant cette question dans John D. Dryzek, Deliberative Democracy and Beyond, Oxford University Press, 2000. 
délibération elle-même. Il ne s'agit pas simplement de persuader les autres, de les convertir à nos préférences, mais de raisonner ensemble, de créer ainsi une communauté politique en acte, qui ne se résume pas en une addition de votes particuliers.

\subsection{La justice internationale}

Une justification des normes internationales de justice est-elle alors possible ? Peut-elle être le modèle de l'inclusion des pays les plus pauvres dans le débat international sur les normes et leur réalisation ? Il existe déjà un embryon d'espace public international, au sens de Habermas. C'est lui qui peut devenir le fer de lance de la démocratisation, et donc de la globalisation des normes de justice.

La délibération, en effet, a des vertus transformatrices. Elle ne se contente pas d'additionner les préférences des électeurs (conception " agrégative »), mais elle vise à les transformer. Un tel projet est très important pour la conception de la justice internationale que nous cherchons à esquisser ici. C'est seulement ainsi que les normes internationales seront perçues non comme une émanation de la volonté et de la culture des pays les plus riches et puissants, mais comme le résultat d'une consultation démocratique à laquelle ils peuvent reconnaitre une vraie légitimité. Le processus de consultation et de délibération peut amorcer une transformation des croyances et des convictions traditionnelles, des " préférences » et créer ainsi les conditions de la paix globale. N'étant le résultat ni d'une conception du bien dominante ni d'une loi naturelle problématique, ces normes peuvent prétendre à une véritable autorité morale.

En élargissant la sphère du politique à l'espace public global de communication, le modèle délibératif répond aux attentes de reconnaissance des citoyens, en particulier de ceux qui viennent de nations défavorisées et appauvries. En permettant l'inclusion dans le processus de prise de décision du plus grand nombre possible de citoyens (ou de membres de la société) grâce à la délibération publique, il entraîne une stabilité véritable, basée sur la participation. Nous serions en présence d'un modèle alternatif de légitimation qui serait la source d'une plus grande stabilité. La délibération est, à différents niveaux de gouvernement et dans des contextes politiques variés, le moyen le plus légitime de résoudre les conflits de principes sur des questions de justice social et la prise de décision qui résulte de la délibération entre citoyens libres et égaux est la forme de justification la plus défendable.

Pour Rawls, comme pour Habermas, c'est donc la délibération qui, seule permet de garantir que le résultat du processus de choix des principes ou de décision politique sera admis comme juste et légitime par des populations divisées par leur culture, leur niveau de vie, leurs régime politiques, etc. Les droits de l'homme et l'idée de justice n'ont d'universalité et de crédibilité que grâce à la procédure de justification, au respect qu'elle témoigne pour la diversité des points de vue et des personnes, à son absence de dogmatisme, à son humilité en quelque sorte. C'est 
la thèse de Habermas dans Droit et démocratie: c'est le droit à la discussion qui fonde les droits humains et les légitime. ${ }^{24}$

Mais la délibération n'est pas un pur processus intellectuel. Elle est solidaire de l'existence d'une communauté de justification. C'est celle-ci qui est cruellement absente de la scène internationale. À la différence d'une communauté de croyances ou de convictions, elle dépasse les limites de l'arbitraire et ne peut être accusée d'impérialisme. Quelles en seraient alors les conditions idéales?

Le caractère public des échanges doit être garanti : nul ne peut être empêché de participer au débat sauf s'il refuse d'en respecter les règles. Il doit y avoir égalité des droits de communication : les participants doivent avoir les mêmes chances de contribuer et de se faire entendre. La condition idéale de véracité s'applique : les participants doivent penser ce qu'ils disent. Enfin, les participants doivent être libres d'intervenir : aucune contrainte interne ou externe ne doit être exercée sur eux.

Il faut compléter ces conditions idéales du débat démocratique par les conditions de réalisation suivantes.

Tout d'abord il doit exister une communauté de justification sur le plan international, et pas seulement des institutions internationales. C'est là le déficit le plus visible de l'organisation que nous connaissons actuellement et dont les décisions ne peuvent prétendre ni à la crédibilité ni à la légitimité en l'absence d'instances de délibération. Seul l'affrontement semble possible. Pour le moment, le forum public est uniquement composé d'États qui font entendre leurs "raisons publiques ", mais qui, en majorité, ne représentent pas les besoins et les droits de leurs populations. À côté des États, il faudrait donc faire de la place pour d'autres partenaires, représentatifs certes, mais émanant de la société civile et capables de transmettre et d'exprimer d'autres raisons ou motifs. Ainsi seulement les normes internationales deviendront véritablement éthiques et globales.

Ensuite, le caractère distinct des identités nationales et culturelles doit être respecté, conformément au principe du pluralisme. De même que les personnes sont des réalités distinctes, ayant chacune leur conception de la vie bonne, de même les cultures et les peuples, au sens sociologique, doivent être respectés dans leur caractère distinctif et leur demande de dignité. Cela n'est possible qu'à condition d'éviter le sophisme essentialiste qui fige les cultures dans des normes et croyances statiques. Mais il faut également éviter l'illusion que les valeurs politiques sont indépendantes de la diversité culturelle et étendre cette reconnaissance du pluralisme aux principes politiques eux-mêmes, ce qui est extrêmement difficile. ${ }^{25}$

24 Habermas, Droit et démocratie, chap. III et IV.

C'est ce que Rawls tente de faire dans Le droit des gens en proposant des principes politiques indépendants du libéralisme et de la culture occidentale, mais sans vraiment y parvenir. Comparer avec David Held et sa conception du "bien démocratique ", à la fois politique et universel. En d'autres termes, il faut en finir avec l'illusion de la "neutralité » des principes politiques. Voir ma critique du républicanisme " universel " dans "Citoyenneté multiculturelle et politique de la reconnaissance », Rue Descartes, 2002. 
Enfin l'institutionnalisation des principes de justice dans un système mondial du droit est indispensable et nous sommes les témoins d'un mouvement dans cette direction avec la création d'un Tribunal pénal international l'an dernier. Comme y insiste Habermas dans Droit et démocratie, sans transformations des constitutions et des législations, sans ratifications des déclarations par les États, les droits des citoyens en matière de justice internationale : droit à la discussion, droit à la participation au processus de production et d'application des normes, resteront lettre morte.

\section{Conclusion}

Nous avons donc tenté de montrer que sans l'établissement d'une véritable communauté de justification, en lieu et place du présent système international, communauté qui soit capable de vraiment démocratiser la société civile internationale, les normes de justice restent une fiction. À partir des travaux de Rawls et de Habermas en matière de théorie de la justice et de théorie de la démocratie, nous avons soutenu que les Déclarations des droits de l'homme ne peuvent fonctionner comme normes correctives et évaluatives que si elles apparaissent justifiées à ceux qui en sont les destinataires. Pour cela il faut qu'ils aient participé au processus d'élaboration et de justification de ces normes. Ce processus doit être une procédure publique qui traite tous les participants de manière égale, qui respecte leur autonomie en tant que sujets de droits, ayant une identité morale, et capables, soit comme États soit comme individus, de contribuer au débat public international.

Cette procédure de justification permettrait non seulement de surmonter l'accusation d'impérialisme moral et culturel que nous évoquions pour commencer, de résoudre donc le paradoxe sur lequel nous nous sommes longuement étendu, de l'imposition non démocratique de normes de justice, mais elle transformerait la société civile internationale, celle du marché et des échanges économiques et culturels, en un espace vraiment public et vraiment global. C'est pourquoi, aussi importante que la communauté de justification, une politique de la reconnaissance est nécessaire de manière à ce que l'éthique de la discussion soit fondée sur une égalité réelle et non pas formelle des partenaires de la justice internationale.

\section{Références}

AUDARD, Catherine. Anthologie de l'utilitarisme, Paris, Presses universitaires de France, 1999. "Citoyenneté multiculturelle et politique de la reconnaissance " in Rue Descartes, nํ 37, Paris, Presses universitaires de France, 2002.

BEITZ, Charles. "Rawls' Law of Peoples », Ethics, vol. 110, n 4, Chicago, University of Chicago Press, 2000 .

DELMAS-MARTY, Mireille. Trois défis pour un droit mondial, Paris, Le Seuil, 1998.

HABERMAS, Jürgen. Droit et Démocratie, trad. Rainer Rochlitz, Paris, Gallimard, 1997. 
HARDT, M. et NEGRI, A. Empire, Cambridge, MA, Harvard University Press, 2000

HELD, David. Democracy and the Global Order, Cambridge, Polity Press, 1995.

HINDESS, Barry. " The Globalization of Citizenship ", à paraitre dans Challenging Citizenship, Colloque de l'Université de Singapour, 2003.

HUME, David. Traité de la nature humaine, III, La morale, trad. P. Saltel, Paris, Garnier-Flammarion, 1993.

KANT, Emmanuel. Vers la paix perpétuelle, trad. J.-F. Poirier et Françoise Proust, Paris, GarnierFlammarion, 1991.

LOCKE, John. Traité du gouvernement civil, trad., Paris, Garnier-Flammarion, 1984.

NUSSBAUM, Martha. Women and Human Development, Cambridge, Cambridge University Press, 2000.

POGGE, Thomas. Realizing Rawls, Cornell University Press, 1989.

RAWLS, John. Théorie de la justice, trad. De Catherine Audard, Paris, Le Seuil, 1987.

$$
\text { . Le droit des gens, trad. de Bertrand Guillarme, Paris, Esprit, } 1996 .
$$

SEN, Amartya, Éthique et économie, trad. Sophie Marnat, Paris, Presses Universitaires de France, 1993. 